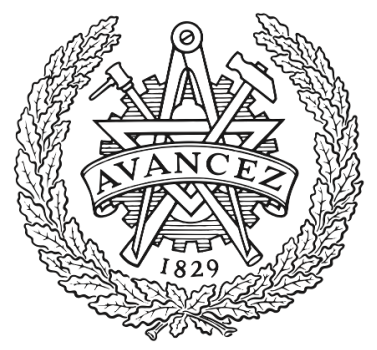

CHALMERS

UNIVERSITY OF TECHNOLOGY

\title{
Enhanced high-frequency performance of top-gated graphene FETs due to substrate-induced improvements in charge carrier saturation velocity
}

Downloaded from: https://research.chalmers.se, 2023-04-26 15:18 UTC

Citation for the original published paper (version of record):

Asad, M., Jeppson, K., Vorobiev, A. et al (2021). Enhanced high-frequency performance of top-gated graphene FETs due to substrate-induced improvements in charge carrier saturation velocity. IEEE Transactions on Electron Devices, 68(2): 899-902. http://dx.doi.org/10.1109/TED.2020.3046172

N.B. When citing this work, cite the original published paper.

(C2021 IEEE. Personal use of this material is permitted.

However, permission to reprint/republish this material for advertising or promotional purposes 


\title{
Enhanced high-frequency performance of top-gated graphene FETs due to substrate-induced improvements in charge carrier saturation velocity
}

\author{
M. Asad, Student Member, IEEE, K. Jeppson, Life Senior Member, IEEE, A. Vorobiev, Member, IEEE, M. \\ Bonmann, Student Member, IEEE, and J. Stake, Senior Member, IEEE
}

\begin{abstract}
High-frequency performance of top-gated graphene field-effect transistors (GFETs) depends to a large extent on the saturation velocity of the charge carriers, a velocity limited by inelastic scattering by surface optical phonons from the dielectrics surrounding the channel. In this work, we show that by simply changing the graphene channel surrounding dielectric with a material having higher optical phonon energy, one could improve the transit frequency and maximum frequency of oscillation of GFETs. We fabricated GFETs on conventional $\mathrm{SiO}_{2} / \mathrm{Si}$ substrates by adding a thin $\mathrm{Al}_{2} \mathrm{O}_{3}$ interfacial buffer layer on top of $\mathrm{SiO}_{2} / \mathrm{Si}$ substrates, a material with about $30 \%$ higher optical phonon energy than that of $\mathrm{SiO}_{2}$, and compared performance with that of GFETs fabricated without adding the interfacial layer. From S-parameter measurements, a transit frequency and a maximum frequency of oscillation of $43 \mathrm{GHz}$ and $46 \mathrm{GHz}$, respectively, were obtained for GFETs on $\mathrm{Al}_{2} \mathrm{O}_{3}$ with $\mathbf{0 . 5} \mu \mathrm{m}$ gate length. These values are approximately $\mathbf{3 0} \%$ higher than those for state-of-theart GFETs of the same gate length on $\mathrm{SiO}_{2}$. For relating the improvement of GFET high-frequency performance to improvements in the charge carrier saturation velocity, we used standard methods to extract the charge carrier velocity from the channel transit time. A comparison between two sets of GFETs with and without the interfacial $\mathrm{Al}_{2} \mathrm{O}_{3}$ layer showed that the charge carrier saturation velocity had increased to $2 \cdot 10^{7} \mathrm{~cm} / \mathrm{s}$ from $1.5 \cdot 10^{7} \mathrm{~cm} / \mathrm{s}$.
\end{abstract}

Index Terms-Field-effect transistors, graphene, transit frequency, maximum frequency of oscillation, optical phonons, saturation velocity.

\section{INTRODUCTION}

Graphene with its high intrinsic charge carrier mobility and velocity [1], [2] has received interest as a channel material for high-frequency field-effect transistors [3]-[6]. Promising transit and maximum oscillation frequencies have been reported for top-gated graphene field-effect transistors (GFETs) yielding competitive frequency times gate length $(f \times L)$ figures of

This project has received funding from the European Union's Horizon 2020 research and innovation programme under grant agreement No 785219 and 881603 and the Swedish Foundation for Strategic Research (Grant No SE13-0061), and the Swedish Research Council (Grant No 2017-04504).

The authors are with Chalmers University of Technology, Department of Microtechnology and Nanoscience, SE-412 96 Gothenburg, Sweden (e-mail: asadmu@chalmers.se). merit [5], [7], [8]. However, the high frequency performance of GFETs, in particular, the maximum frequency of oscillation $\left(f_{\max }\right)$, is limited by the intrinsically high drain conductance $\left(g_{\mathrm{d}}\right)$, caused by zero-bandgap in monolayer graphene, and extrinsic factors such as the elastic and inelastic scattering of charge carriers associated with dielectric materials surrounding the graphene channel [6], [9]-[11].

Charge carrier saturation velocity $\left(v_{\text {sat }}\right)$ of conventional GFETs fabricated on $\mathrm{SiO}_{2} /$ silicon substrates is limited by inelastic scattering of the low energy surface optical phonons of the adjacent dielectrics [12]-[15]. The optical phonon energy $\left(\hbar w_{\mathrm{op}}\right)$ of $\mathrm{SiO}_{2}$, the commonly used substrate dielectric, is in the range of 50-60 meV, which is much less than the 160-200 $\mathrm{meV}$ of graphene and will therefore limit the charge carrier velocity in graphene [13], [16]. GFETs with the graphene channel embedded between a $\mathrm{SiO}_{2}$ substrate dielectric and $\mathrm{Al}_{2} \mathrm{O}_{3}$ gate dielectric exhibit a saturation velocity of $1.5 \cdot 10^{7}$ $\mathrm{cm} / \mathrm{s}$ [15]. The hexagonal boron nitride $(\mathrm{hBN})$ with optical phonon energy around $100 \mathrm{meV}$ [4] is a promising option, but not yet feasible for wafer scale device manufacturing. A possible option is to completely surround the graphene channel with layers of $\mathrm{Al}_{2} \mathrm{O}_{3}$, a dielectric with relatively high $\hbar w_{\mathrm{op}}=87$ $\mathrm{meV}$ [14], for application in high frequency FETs [17], [18].

In this letter, we show the improvements in the saturation velocity and corresponding enhancement in GFET highfrequency performance by measuring GFETs with a thin buffer layer of $\mathrm{Al}_{2} \mathrm{O}_{3}$ on the $\mathrm{SiO}_{2} / \mathrm{Si}$ substrate [18], [19]. We measured the extrinsic transit frequency $\left(f_{\mathrm{T}}\right)$ and $f_{\max }$ up to $43 \mathrm{GHz}$ and $46 \mathrm{GHz}$, respectively, for $0.5 \mu \mathrm{m}$ channel length, which outperform state-of-the-art GFETs of similar gate lengths. The improvements in the high frequency performance is correlated with a corresponding $30 \%$ increase in the $v_{\text {sat }}$, up to $2 \cdot 10^{7} \mathrm{~cm} / \mathrm{s}$, in GFET with $\mathrm{Al}_{2} \mathrm{O}_{3}$ buffer layer.

\section{Methods}

A set of GFETs was fabricated using the same design and fabrication process as previously described [18], the only exception being that a $60 \mathrm{~nm}$ thick $\mathrm{Al}_{2} \mathrm{O}_{3}$ layer was added by atomic layer deposition on top of the $1 \mu \mathrm{m}$ thick thermally grown $\mathrm{SiO}_{2}$ layer covering the high-resistivity silicon 


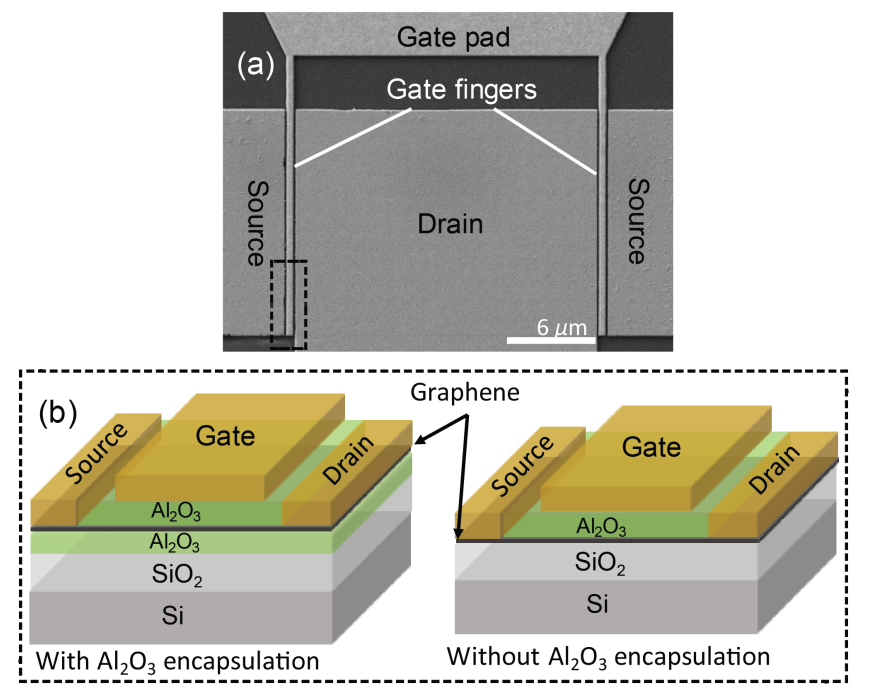

Fig. 1. (a) Top view SEM image of a fabricated two-finger GFET, and (b) cross-sectional schematic views of GFET gate region, corresponding to the dashed box in (a), with and without $\mathrm{Al}_{2} \mathrm{O}_{3}$ buffer layer. NB! Drawings are not to scale.

substrate. After graphene transfer to the $\mathrm{Al}_{2} \mathrm{O}_{3} / \mathrm{SiO}_{2} /$ silicon substrate, a $22 \mathrm{~nm}$ top gate $\mathrm{Al}_{2} \mathrm{O}_{3}$ dielectric was deposited leaving the graphene channel interfacing $\mathrm{Al}_{2} \mathrm{O}_{3}$ both above and below as shown in Fig. 1. Also shown in Fig. 1 is a GFET from the reference set of devices with its graphene channel placed directly on the substrate $\mathrm{SiO}_{2}$ layer. The gate geometries of the two sets of two-finger GFETs, which we used for comparison, were $0.5 \mu \mathrm{m}$ for the gate length and $2 \times 15 \mu \mathrm{m}$ for the gate width, see an SEM image of the twofinger GFET in Fig. 1.

After fabrication, GFETs from both sets of devices were characterized for extracting their low-field dc-parameters, and for finding the $f_{\mathrm{T}}$ and $f_{\max }$. Fig. 2 shows the drain resistances vs. gate voltage, and the output characteristics ( $I_{\mathrm{D}}$ vs. $\left.V_{\mathrm{D}}\right)$ of two selected GFETs with similar low-field mobility, one from each set, measured at room temperature. Also shown in Fig. 2(b) is the intrinsic output conductance obtained from $g_{\mathrm{d}}=\partial I_{\mathrm{D}} / \partial\left(V_{\mathrm{D}}-I_{\mathrm{D}} R_{\mathrm{C}}\right)$ [20], where $R_{\mathrm{C}}$ is the contact resistance and corrected as Chou et al. [21]. By fitting the drain resistance models [22], [23] to the experimental data, we found that the residual charge carrier concentrations $\left(n_{0}\right)$, low-field mobility $\left(\mu_{0}\right)$, voltage overdrive $\left(V_{\mathrm{GS}}-V_{\text {Dirac }}\right)$ and $R_{\mathrm{C}}$ are $12 \times 10^{11} \mathrm{~cm}^{-2}, 1800 \mathrm{~cm}^{2} / \mathrm{Vs}, 20 \Omega, 0.7 \mathrm{~V}$ and $20 \times 10^{11} \mathrm{~cm}^{-2}$, $1800 \mathrm{~cm}^{2} / \mathrm{Vs}, 10.5 \Omega, 0.8 \mathrm{~V}$ in the two selected GFETs with and without $\mathrm{Al}_{2} \mathrm{O}_{3}$, respectively.

The GFET S-parameters were measured using an Agilent N5230A VNA and calibrated using standard two-port SOLT calibrations. The S-parameters were used to obtain the smallsignal current gain $\left(h_{21}\right)$, and the unilateral power gain $(U)$ from which the extrinsic transit frequency $f_{\mathrm{T}}$ and the extrinsic maximum frequency of oscillation $f_{\max }$ could be extracted at $0 \mathrm{~dB}$ [24], [25]. Some typical plots of the $h_{21}$ vs. frequency, and the transit frequency vs. drain voltage are shown in Fig. 3 for the two selected GFETs. In the analysis below, the charge carrier velocity was extracted directly from intrinsic transit
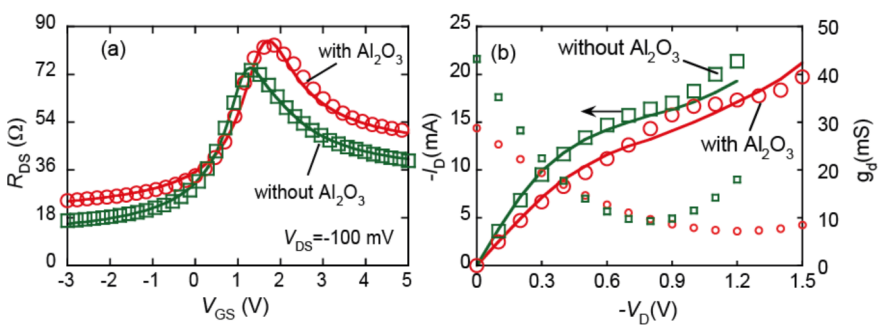

Fig. 2. DC-characteristics of two GFETs with and without $\mathbf{A l}_{\mathbf{2}} \mathbf{O}_{3}$ buffer layer. (a) Drain resistance vs. gate voltage. Solid lines show models [22], [23] fitted to experimental data (symbols). (b) Drain current and intrinsic drain conductance vs. drain voltage. The lines are simulations using the models presented by Meric et al. [4] including the thermally generated charge carriers as in Dorgan et al. [12].

frequency $f_{\mathrm{T}, \text { int }} \approx v / 2 \pi L$, where $v$ is the charge carrier velocity, using delay time analysis [26], [27].

\section{Results And Discussions}

In this analysis, GFETs from two sets of samples with and without $\mathrm{Al}_{2} \mathrm{O}_{3}$ buffer layer were characterized and their high frequency performance compared as function of low-field mobility, as shown in Fig. 4(a). The low-field mobility has been chosen as the most appropriate indicator of the graphene quality enabling a fair comparison between the two sets of devices [18]. Despite variations in the obtained data, there is a clear improvement in the transit frequency of GFETs with $\mathrm{Al}_{2} \mathrm{O}_{3}$ buffer layer as compared to the GFETs with graphene on $\mathrm{SiO}_{2} / \mathrm{Si}$ substrate. At low-field mobilities around 2000 $\mathrm{cm}^{2} / \mathrm{Vs}$, GFETs with $\mathrm{Al}_{2} \mathrm{O}_{3}$ graphene channel encapsulation show transit frequencies at least $10 \mathrm{GHz}$ higher than GFETs without. A similar behaviour was observed for the maximum frequency of oscillation.

We show below, by applying the delay time analysis, that the improvement in the high performance is related to the increase in the charge carrier velocity. As it can be seen from Fig. 3(b), the GFET with $\mathrm{Al}_{2} \mathrm{O}_{3}$ buffer layer has a maximum $f_{\mathrm{T}}$ of 43 $\mathrm{GHz}$, while the GFET without the $\mathrm{Al}_{2} \mathrm{O}_{3}$ buffer layer has $f_{\mathrm{T}}$ up to $34 \mathrm{GHz}$. Next step is to relate the experimentally measured extrinsic $f_{\mathrm{T}}=1 /\left(2 \pi \tau_{\text {tot }}\right)$, where $\tau_{\text {tot }}$ is the total delay time, to the intrinsic transit frequency $f_{\mathrm{T}, \text { int }}=1 /\left(2 \pi \tau_{\text {int }}\right)$. It can be done applying the analysis of the FET small-signal equivalent circuit pioneered by Tasker and Hughes [28], and later used in [8], [26], [27] as:

$$
f_{T}=\frac{f_{T, i n t}}{1+g_{d} R_{C}+\frac{C_{g d} \cdot g_{m} \cdot R_{C}}{C_{g s}+C_{g d}}+\frac{C_{p a d}}{C_{g s}+C_{g d}}} .
$$

In this equation, $g_{\mathrm{m}}$ is the intrinsic transconductance, $C_{\mathrm{gs}}, C_{\mathrm{gd}}$ and $C_{\text {pad }}$ are the gate-source, gate-drain and parasitic gate pad capacitances, respectively, and $R_{\mathrm{C}}=R_{\mathrm{S}}+R_{\mathrm{D}}$ is the sum of the source and drain contact resistances. $R_{\mathrm{C}}$ is obtained from the $R_{\mathrm{DS}}$ vs. $V_{\mathrm{GS}}$ characteristic. For the GFET gate capacitance we assumed $C_{\mathrm{gs}}=W L C_{\mathrm{ox}} / 2$. Here, $C_{\mathrm{ox}}=\epsilon_{0} \epsilon_{\mathrm{ox}} / t_{\mathrm{ox}}=3 \mathrm{mF} / \mathrm{m}^{2}$ where $\epsilon_{0}$ is the permitivity of free space, $\epsilon_{\mathrm{ox}}$ is the permittivity and $t_{\mathrm{ox}}$ is the thickness of the gate oxide, because of the ambipolarity of the channel at high field, we assume that $C_{\mathrm{gd}}=k C_{\mathrm{gs}}$, where $k$ used as a fitting parameter. It can be shown that the effects of the capacitance coupling between $C_{\mathrm{gs}}, C_{\mathrm{gd}}$ 

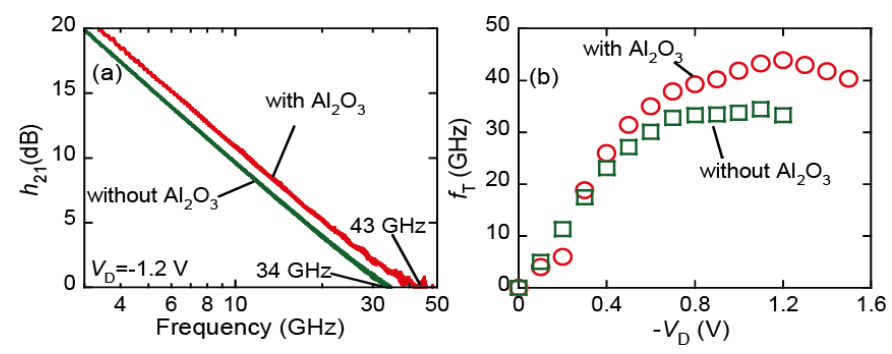

Fig. 3. High frequency performance of GFETs with and without $\mathrm{Al}_{2} \mathrm{O}_{3}$ buffer layer. (a) Small-signal current gain $\left(\boldsymbol{h}_{21}\right)$ vs. frequency. (b) Transit frequency $\left(\boldsymbol{f}_{\mathrm{T}}\right)$ vs. drain voltage.

and $C_{\text {pad }}$ can be ignored [29]. A gate pad capacitance $C_{\text {pad }}$ of $8 \mathrm{fF}$ was obtained as described in Numila et al. [26].

From eq. (1), the transit time was easily found by using $\tau_{\text {int }}=\left(C_{g s}+C_{g d}\right) / g_{\mathrm{m}}$, and the $g_{\mathrm{d}}$ values shown in Fig. 2(b). The $g_{\mathrm{m}}$ obtained here for GFET with $\mathrm{Al}_{2} \mathrm{O}_{3}$ buffer layer is $16 \mathrm{~mA} / \mathrm{V}$ compare to $12 \mathrm{~mA} / \mathrm{V}$ for the GFET without $\mathrm{Al}_{2} \mathrm{O}_{3}$ interface dielectric. Then, the charge carrier velocity was calculated using the found intrinsic transit time. Fig. 4(b) shows the effective charge carrier velocity versus drain field $E_{\text {int }}=\left(V_{\mathrm{D}}-R_{\mathrm{C}} I_{\mathrm{D}}\right) / L$ for the two selected GFETs. In both GFETs the charge carrier velocity saturated at drain fields above $10 \mathrm{kV} / \mathrm{cm}$, which is in good agreement with previous results [12], [15]. Using the Caughey-Thomas velocity model [30], a $v_{\text {sat }}$ of $2.0 \cdot 10^{7} \mathrm{~cm} / \mathrm{s}$ was found for the GFET with $\mathrm{Al}_{2} \mathrm{O}_{3}$ buffer dielectric, which is approximately $30 \%$ higher than the $1.5 \cdot 10^{7} \mathrm{~cm} / \mathrm{s}$ found for GFETs without $\mathrm{Al}_{2} \mathrm{O}_{3}$ buffer dielectric. Fig. 4(b) also shows the semi-empirical velocity model (solid lines) fitted to experimental data using $\gamma$ as a fitting parameter. Also, Fig. 4(b) shows the theoretically estimated saturation velocities using the model by Dorgan et al., assuming that $v_{\text {sat }}$ is limited by inelastic emission of optical phonons [12]. These values were calculated using a carrier density obtained from the output characteristic using models [4], [12] at high field for both GFETs with and without $\mathrm{Al}_{2} \mathrm{O}_{3}$ buffer dielectric.

Combining the analysis of theory and experimental data, one can conclude that charge carrier velocity in the samples with $\mathrm{Al}_{2} \mathrm{O}_{3}$ buffer interface layer is improved due to the higher optical phonon energy and, partly, due to the lower carriers concentration compared to the sample without $\mathrm{Al}_{2} \mathrm{O}_{3}$ buffer layer. Our analysis of other devices indicates that this conclusion is valid in the whole studied range of the lowfield mobilities. According to our analysis, the GFET without $\mathrm{Al}_{2} \mathrm{O}_{3}$ buffer layer, in spite of lower saturation velocity, reveal higher drain current (Fig. 2(b)), because of higher intrinsic drain voltage and, partly, because of higher total concentration of the charge carriers.

Finally, as it can be seen from Fig. 5, the highest extrinsic $f_{\text {max }}$ for GFETs with and without $\mathrm{Al}_{2} \mathrm{O}_{3}$ buffer layer were measured to be $46 \mathrm{GHz}$ and $37 \mathrm{GHz}$, respectively. These values are the highest reported so far for GFETs of similar gate lengths [8]. Fig. 5 shows two dependencies of GFETs with $\mathrm{Al}_{2} \mathrm{O}_{3}$ buffer layer. The gain of one GFET with $\mathrm{Al}_{2} \mathrm{O}_{3}$ buffer layer is higher of that without $\mathrm{Al}_{2} \mathrm{O}_{3}$ buffer layer across whole frequency range. The reduced gain of one GFET at lower
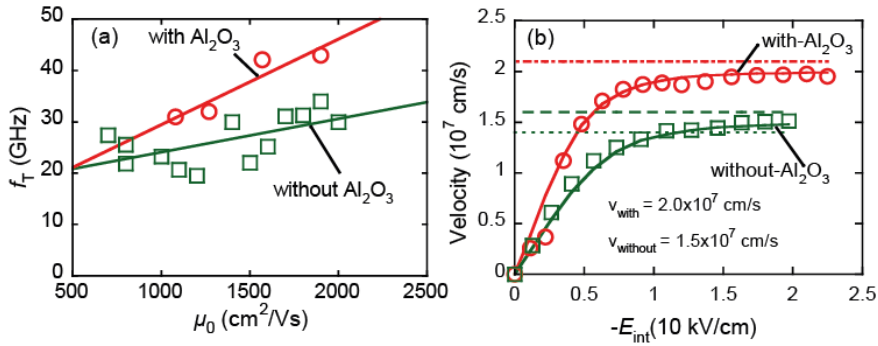

Fig. 4. Effects of $\mathbf{A l}_{2} \mathbf{O}_{3}$ buffer layer on GFET performance. (a) Transit frequency $\left(\boldsymbol{f}_{\mathbf{T}}\right)$ vs. low-field mobility $\left(\boldsymbol{\mu}_{\mathbf{0}}\right)$ for GFETs with and without $\mathrm{Al}_{2} \mathrm{O}_{3}$ buffer layer. Solid lines represent linear trendlines. (b) Charge carrier velocity vs. electric field for two GFETs $\left(\mu_{0}=1800 \mathrm{~cm}^{2} / \mathrm{Vs}\right)$ with and without $\mathrm{Al}_{2} \mathrm{O}_{3}$ buffer layer. Solid lines show the CaugheyThomas model [30] fitted to experimentally extracted data (symbols), while dotted line and dashed lines show the theoretically estimated saturation velocities [12], using $\hbar \boldsymbol{w}_{\mathrm{op}}=60 \mathrm{meV}, n=\mathbf{2 4} \times \mathbf{1 0}^{\mathbf{1 1}} \mathrm{cm}^{-2}$ and $n=17 \times 10^{11} \mathrm{~cm}^{-2}$, respectively. Also shown dashed dotted line corresponds to $\hbar \boldsymbol{w}_{\mathrm{op}}=87 \mathrm{meV}, \boldsymbol{n}=\mathbf{1 7} \times \mathbf{1 0}^{\mathbf{1 1}} \mathrm{cm}^{-\mathbf{2}}$. The carrier densities obtained from output characteristics at $\boldsymbol{E}_{\text {int }}=15 \mathrm{kV} / \mathrm{cm}$.

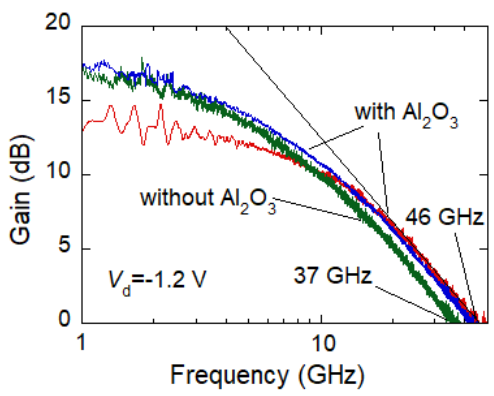

Fig. 5. Maximum frequency of oscillation: unilateral power gain $(\boldsymbol{U})$ vs. frequency of GFETs with and without $\mathrm{Al}_{2} \mathrm{O}_{3}$ buffer layer.

frequencies might be attributed to measurement artifact and/or to higher concentration of traps in the gate stack [31]. In terms of scaling, the GFETs in this study have a high $f_{\max } \times L$ figure of merit of $23 \mathrm{GHz} \cdot \mu \mathrm{m}$, a value comparable to some of the best published MOSFET values [32]. A possible road ahead for further improving GFET high-frequency performance might be to use dielectric materials with even higher optical phonon energies. Preliminary estimates suggest that the $v_{\text {sat }}$ in GFETs encapsulated by hBN could be as high as $5 \cdot 10^{7} \mathrm{~cm} / \mathrm{s}$ corresponding to a $f_{\mathrm{T}, \text { int }}$ of $400 \mathrm{GHz}$ at $200 \mathrm{~nm}$ channel lengths.

\section{CONClusion}

In summary, high-frequency top-gated graphene field-effect transistors were fabricated on $\mathrm{Al}_{2} \mathrm{O}_{3}$ substrate dielectrics, and their performance was compared to that of GFETs on $\mathrm{SiO}_{2}$, a dielectric with a lower $\hbar w_{\text {op }}$. High extrinsic transit and maximum oscillation frequencies of $43 \mathrm{GHz}$ and $46 \mathrm{GHz}$, respectively, were obtained experimentally (indicating a figure of merit of $\left.f_{\max } \times L=23 \mathrm{GHz} \cdot \mu \mathrm{m}\right)$. The improvement found compared to GFETs on $\mathrm{SiO}_{2}$ can be explained mainly by the higher optical phonon energy of the encapsulating material resulting in a higher charge carrier saturation velocity. In addition to scaling of the gate length, one road ahead for further progress in high-frequency GFET performance might 
be to use the substrate and gate dielectrics with even higher optical phonon energies.

\section{REFERENCES}

[1] L. Banszerus, M. Schmitz, S. Engels, J. Dauber, M. Oellers, F. Haupt, K. Watanabe, T. Taniguchi, B. Beschoten, and C. Stampfer, "Ultrahigh-mobility graphene devices from chemical vapor deposition on reusable copper," Sci. adv., vol. 1, no. 6, p. e1500222, 2015, doi:10.1126/sciadv.1500222.

[2] M. A. Yamoah, W. Yang, E. Pop, and D. Goldhaber-Gordon, "High-velocity saturation in graphene encapsulated by hexagonal boron nitride," ACS nano, vol. 11, no. 10, pp. 9914-9919, 2017, doi:10.1021/acsnano.7b03878.

[3] M. C. Lemme, T. J. Echtermeyer, M. Baus, and H. Kurz, "A graphene field-effect device," IEEE Electron Device Lett., vol. 28, no. 4, pp. 282-284, 2007, doi:10.1109/LED.2007.891668.

[4] I. Meric, M. Y. Han, A. F. Young, B. Ozyilmaz, P. Kim, and K. L. Shepard, "Current saturation in zero-bandgap, top-gated graphene fieldeffect transistors," Nat. nanotechnol., vol. 3, no. 11, pp. 654-659, 2008, doi:10.1038/nnano.2008.268.

[5] Y.-M. Lin, C. Dimitrakopoulos, K. A. Jenkins, D. B. Farmer, H.-Y. Chiu, A. Grill, and P. Avouris, "100-ghz transistors from wafer-scale epitaxial graphene," Science, vol. 327, no. 5966, pp. 662-662, 2010, doi:10.1126/science.1184289.

[6] F. Schwierz, "Graphene transistors: status, prospects, and problems," Proceedings of the IEEE, vol. 101, no. 7, pp. 1567-1584, 2013, doi:10.1109/JPROC.2013.2257633.

[7] Z. Guo, R. Dong, P. S. Chakraborty, N. Lourenco, J. Palmer, Y. Hu, M. Ruan, J. Hankinson, J. Kunc, J. D. Cressler et al., "Record maximum oscillation frequency in c-face epitaxial graphene transistors," Nano letters, vol. 13, no. 3, pp. 942-947, 2013, doi:10.1021/n1303587r.

[8] M. Bonmann, M. Asad, X. Yang, A. Generalov, A. Vorobiev, L. Banszerus, C. Stampfer, M. Otto, D. Neumaier, and J. Stake, "Graphene Field-Effect Transistors With High Extrinsic $f_{T}$ and $f_{\text {max }}$," IEEE Electron Device Lett., vol. 40, no. 1, pp. 131-134, 2018, doi:10.1109/LED.2018.2884054.

[9] J. Lee, H.-J. Chung, J. Lee, H. Shin, J. Heo, H. Yang, S.-H. Lee, S. Seo, J. Shin, U.-i. Chung et al., "Rf performance of prepatterned locally-embedded-back-gate graphene device," in $2010 \mathrm{In}$ ternational Electron Devices Meeting. IEEE, 2010, pp. 23-5, doi:10.1109/IEDM.2010.5703422.

[10] L. Liao, Y.-C. Lin, M. Bao, R. Cheng, J. Bai, Y. Liu, Y. Qu, K. L. Wang, Y. Huang, and X. Duan, "High-speed graphene transistors with a self-aligned nanowire gate," Nature, vol. 467, no. 7313, pp. 305-308, 2010, doi:10.1038/nature09405.

[11] Y. Wu, Y.-m. Lin, A. A. Bol, K. A. Jenkins, F. Xia, D. B. Farmer, Y. Zhu, and P. Avouris, "High-frequency, scaled graphene transistors on diamond-like carbon," Nature, vol. 472, no. 7341, pp. 74-78, 2011, doi:10.1038/nature09979

[12] V. E. Dorgan, M.-H. Bae, and E. Pop, "Mobility and saturation velocity in graphene on $\mathrm{SiO}_{2}$," Appl. Phys. Lett., vol. 97, no. 8, p. 082112, 2010, doi:10.1063/1.3483130.

[13] J. Chauhan and J. Guo, "High-field transport and velocity saturation in graphene," Appl. Phys. Lett., vol. 95, no. 2, p. 023120, 2009, doi:10.1063/1.3182740.

[14] J. Chauhan and J. Guo, "Inelastic phonon scattering in graphene FETs," IEEE trans. on electron devices, vol. 58, no. 11, pp. 3997-4003, 2011, doi:10.1109/TED.2011.2164253.

[15] M. Bonmann, A. Vorobiev, M. A. Andersson, and J. Stake, "Charge carrier velocity in graphene field-effect transistors," Appl. Phys. Lett., vol. 111 , no. 23, p. 233505 , 2017, doi:10.1063/1.5003684.

[16] M. Fischetti, D. Neumayer, and E. Cartier, "Effective electron mobility in Si inversion layers in MOS systems with a high-k insulator: The role of remote phonon scattering," J. Appl. Phys., vol. 90, no. 9, p. 4587, 2001, doi:10.1063/1.1405826

[17] A. Vorobiev, M. Bonmann, M. Asad, X. Yang, L. Banszerus, C. Stampfer, M. Otto, D. Neumaier, and J. Stake, "Graphene field-effect transistors for millimetre wave amplifiers," Proceeding of the IEEE, 2019, doi:10.1109/IRMMW-THz.2019.8874149.

[18] M. Asad, M. Bonmann, X. Yang, A. Vorobiev, K. Jeppson, L. Banszerus, O. Martin, C. Stampfer, D. Neumaier, and J. Stake, "The dependence of the high-frequency performance of graphene field-effect transistors on channel transport properties," IEEE J. of the Electron Devices Soc., pp. 1-1, 2020, doi:10.1109/JEDS.2020.2988630.
[19] P. C. F. Guerro, F. Pasadas, M. Bonmann, M. Asad, X. Yang, A. Generalov, A. Voroviev, L. Banszerus, C. Stampfer, M. Otto et al., "Does carrier velocity saturation help to enhance $\mathrm{f} \max$ in graphene field-effect transistors?" Nanoscale Advances, 2020, doi:10.1039/C9NA00733D.

[20] F. Schwierz, "Graphene transistors," Nat. nanotechnol., vol. 5, no. 7, p. 487, 2010, doi:10.1038/nnano.2010.89.

[21] S. Y. Chou and D. Antoniadis, "Relationship between measured and intrinsic transconductances of FET's," IEEE Trans. on Electron Devices, vol. 34, no. 2, pp. 448-450, 1987, doi:10.1109/T-ED.1987.22942.

[22] S. Kim, J. Nah, I. Jo, D. Shahrjerdi, L. Colombo, Z. Yao, E. Tutuc, and S. K. Banerjee, "Realization of a high mobility dual-gated graphene field-effect transistor with $\mathrm{Al}_{2} \mathrm{O}_{3}$ dielectric," Appl. Phys. Lett., vol. 94, no. 6, p. 062107, 2009, doi:10.1063/1.3077021.

[23] O. Habibpour, J. Vukusic, and J. Stake, "A large-signal graphene FET model," IEEE Trans. on Electron Devices, vol. 59, no. 4, pp. 968-975, 2012, doi:10.1109/TED.2012.2182675.

[24] S. Mason, "Power gain in feedback amplifier," Transactions of the IRE Professional Group on Circuit Theory, no. 2, pp. 20-25, 1954, doi:10.1109/TCT.1954.1083579.

[25] M. S. Gupta, "Power gain in feedback amplifiers, a classic revisited," IEEE Trans. on Microwave Theory and Tech., vol. 40, no. 5, pp. 864879, 1992, doi:10.1109/22.137392.

[26] K. Nummila, A. Ketterson, and I. Adesida, "Delay time analysis for short gate-length GaAs MESFETs," Solid-state electron., vol. 38, no. 2, pp. 517-524, 1995, doi:10.1016/0038-1101(94)00097-Y.

[27] H. Wang, A. Hsu, D. S. Lee, K. K. Kim, J. Kong, and T. Palacios, "Delay analysis of graphene field-effect transistors," IEEE electron device lett., vol. 33, no. 3, pp. 324-326, 2012, doi:10.1109/LED.2011.2180886.

[28] P. J. Tasker and B. Hughes, "Importance of source and drain resistance to the maximum $f_{\mathrm{T}}$ of millimeter-wave MODFETs," IEEE Electron Device Lett., vol. 10, no. 7, pp. 291-293, 1989, doi:10.1109/55.29656.

[29] M. S. Fuhrer and J. Hone, "Measurement of mobility in dual-gated mos 2 transistors," Nature nanotechnology, vol. 8, no. 3, pp. 146-147, 2013, doi:10.1038/nnano.2013.30.

[30] D. Caughey and R. Thomas, "Carrier mobilities in silicon empirically related to doping and field," Proceedings of the IEEE, vol. 55, no. 12, pp. 2192-2193, 1967, doi:10.1109/PROC.1967.6123.

[31] M. Hellenbrand, E. Lind, O.-P. Kilpi, and L.-E. Wernersson, "Effects of traps in the gate stack on the small-signal of response of iii-v nanowire mosfets," Solid-State Electronics, p. 107840, 2020, doi:10.1016/j.sse.2020.107840.

[32] L. Tiemeijer, H. Boots, R. Havens, A. Scholten, P. De Vreede, P. Woerlee, A. Heringa, and D. Klaassen, "A record high $150 \mathrm{GHz} f_{\max }$ realized at $0.18 \mu \mathrm{m}$ gate length in an industrial RF-CMOS technology," in International Electron Devices Meeting. Technical Digest (Cat. No. 01CH37224). IEEE, 2001, pp. 10-4, doi:10.1109/IEDM.2001.979471. 